\title{
Bir Normal Hemen Hemen Parakontakt Metrik Manifoldun Quasi-Konformal Ĕgrilik Tensörü Üzerine
}

\author{
On the Quasi-Conformal Curvature Tensor of a Normal Almost Paracontact Metric \\ Manifold
}

\section{Ümit YILDIRIM*,a, Mehmet ATÇEKEN ${ }^{\mathrm{b}}$}

Gaziosmanpaşa Üniversitesi, Fen Edebiyat Fakültesi, Matematik, Bölümü, 60500, Tokat

• Geliş tarihi / Received: 11.04.2018 • Düzeltilerek geliş tarihi / Received in revised form: 15.07 .2018 • Kabul tarihi / Accepted: 06.08 .2018

$\ddot{O} \mathbf{z}$

Bu makalede bir normal hemen hemen parakontakt metrik manifoldun $\tilde{C}\left(\xi, U_{1}\right) R=0, \tilde{C}\left(\xi, U_{1}\right) P=0, \tilde{C}\left(\xi, U_{1}\right) \tilde{Z}=0$, $\tilde{C}\left(\xi, U_{1}\right) S=0$ ve $\tilde{C}\left(\xi, U_{1}\right) \tilde{C}=0$, şartlarını sağlaması durumunda ortaya çıkan sonuçlar çalışılmıştır. Bu sonuçlara göre manifold karakterize edilmiştir. Burada $R$, Riemann eğrilik tensörü, $\tilde{C}$, quasi-konformal eğrilik tensörü, $P$, projektif eğrilik tensörü, $\tilde{Z}$, concircular eğrilik tensörü ve $S$ Ricci tensörüdür.

Anahtar kelimeler: Concircular eğrilik tensörü, Einstein manifold, Normal hemen hemen parakontakt metrik manifold, Quasi-konformal eğrilik tensörü

\begin{abstract}
In the present paper, we have studied the curvature tensors of a normal almost paracontact metric manifold satisfying the conditions $\tilde{C}\left(\xi, U_{1}\right) R=0, \tilde{C}\left(\xi, U_{1}\right) P=0, \tilde{C}\left(\xi, U_{1}\right) \tilde{Z}=0, \tilde{C}\left(\xi, U_{1}\right) S=0$ and $\tilde{C}\left(\xi, U_{1}\right) \tilde{C}=0$. According these cases, we classified normal almost paracontact metric manifolds, where $R$ is the Riemanniann curvature tensor, $\tilde{C}$ is the quasi-conformal curvature tensor, $P$ is the projective curvature tensor, $\tilde{Z}$ is the concircular curvature tensor and $S$ is the Ricci tensor.
\end{abstract}

Keywords: Concircular curvature tensor, Einstein manifold, Normal almost paracontact metric manifold, Quasiconformal curvature tensor

*ä Ümit YILDIRIM; umit.yildirim@ gop.edu.tr; Tel: (0543) 37538 15; orcid.org/0000-0002-7178-4223

${ }^{\mathrm{b}}$ orcid.org/0000-0001-8665-5945 


\section{Giriş}

Parakontakt geometri üzerine çalışmalar ilk olarak Sato (1976) ile başlamıştır. Daha sonra Kaneyuki ve Williams (1985), manifoldlar üzreinde parakontakt yapılar için bazı karakterizasyonlar vermişlerdir. Acet vd., (2012; 2016), paraSasakian manifoldlarda kanonik parakontakt konneksiyon için bazı eğrilik şartlarını incelmişlerdir. Ayrıca son yıllarda pek çok yazar, matematiğin yanında fizikte de çok önemli bir yeri olan normal (para) hemen hemen kontakt metrik manifoldların simetri ve eğrilik tensörlerinin özelikleri ile ilgili çalışmalar yapmışlardır (Szabo, 1982; Olszak, 1986; De ve Mondal, 2009; De vd., 2009; Erdem, 2015; Erken, 2015).

Atçeken ve Yıldırım (2016) quasi-konformal eğrilik tensörünü $\quad C(\alpha)$-manifoldlarında, Atçeken (2013) $(L C S)_{n}$-manifoldlarında, De vd. (2008) Sasakian manifoldlarında, De ve Sarkar (2012) $(k, \mu)$-kontakt metrik manifoldlarında, Hosseinzadeh ve Taleshian (2012) $N(k)$-quasiEinstein manifoldlarında incelemişlerdir.

Biz de şimdiye kadar yapılan bu çalışmalar 1şı̆̆ında bir normal hemen hemen parakontakt metrik manifoldunda quasi-konformal eğrilik tensörünün sağladığı bazı eğrilik şartlarını araştırdık ve elde ettiğimiz sonuçlara göre sabit kesit eğrilikli bir normal hemen hemen parakontakt metrik manifold için bazı sinıflandırmalar elde ettik.

\section{Temel Bilgiler}

Tanım 2.1. $M, n-$ boyutlu bir Riemann manifoldu ve $R, M$ Riemann manifoldunun Riemanninan eğrilik tensörü olsun. $\left\{e_{1}, e_{2}, \ldots, e_{n}\right\}$ cümlesi $\chi(M)$ nin ortonormal vektör alanlar1 olmak üzere $\forall U_{1}, U_{2} \in \chi(M)$ için

$$
\begin{gathered}
S: \chi(M) \times \chi(M) \rightarrow C^{\infty}(M, \mathbb{R}) \\
\left(U_{1}, U_{2}\right) \rightarrow S\left(U_{1}, U_{2}\right)= \\
\sum_{i=1}^{n} g\left(R\left(U_{1}, e_{i}\right) e_{i}, U_{2}\right)
\end{gathered}
$$

şeklinde tanımlı $(0,2)$ - tipindeki tensöre $M$ nin Ricci tensörü adı verilir. Kolayca görülebilir ki Ricci tensörü simetriktir. Ayrıca $M$ nin Ricci operatörü $Q$ ise

$$
S\left(U_{1}, U_{2}\right)=g\left(Q U_{1}, U_{2}\right)
$$

şeklinde tanımlıdır.
Tanım 2.2. $M, n-$ boyutlu bir Riemann manifoldu ve $\left\{e_{1}, e_{2}, \ldots, e_{n}\right\}$ lokal ortonormal vektör alanları olmak üzere

$r=\sum_{\mathrm{i}=1}^{\mathrm{n}} S\left(e_{i}, e_{i}\right)$

değerine $M$ nin skaler eğrilik fonsiyonu denir (O’Neill, 1983).

Tanım 2.3. $M, n-$ boyutlu bir Riemann manifoldu olmak üzere $\forall U_{1}, U_{2} \in \chi(M)$ için

$S\left(U_{1}, U_{2}\right)=\lambda g\left(U_{1}, U_{2}\right)$

olacak biçimde $M$ üzerinde bir $\lambda$ fonksiyonu varsa, yani, $M$ nin Ricci tensörü $S$, metrik tensör $g$ nin bir katı ise $M$ ye Einstein manifoldu adı verilir (Boothby, 1986).

Tanım 2.4. $M, n-$ boyutlu bir Riemann manifoldu olsun. Eğer, $S$ Ricci tensörü olmak üzere, $\forall U_{1}, U_{2} \in \chi(M)$ için

$S\left(U_{1}, U_{2}\right)=a g\left(U_{1}, U_{2}\right)+b \eta\left(U_{1}\right) \eta\left(U_{2}\right)$

eşitliği sağlanıyorsa $M$ ye $\eta$-Einstein manifoldu ad1 verilir.

Burada $a$ ve $b M$ üzerinde fonksiyonlar ve $\eta-$ da 1 -formdur (Boothby, 1986).

Tanım 2.5. $(M, g)$ bir Riemann manifoldu olsun. $T_{M}(p)$ tanjant uzayının iki boyutlu alt uzayı $\Pi$ olmak üzere $U_{1}, U_{2} \in \Pi$ tanjant vektörleri için $Q$ fonksiyonu;

$Q\left(U_{1}, U_{2}\right)=g\left(U_{1}, U_{1}\right) g\left(U_{2}, U_{2}\right)-g\left(U_{1}, U_{2}\right)^{2}$

biçiminde tanımlansın. $Q\left(U_{1}, U_{2}\right) \neq 0$ olmak üzere

$K\left(U_{1}, U_{2}\right)=\frac{g\left(R\left(U_{1}, U_{2}\right) U_{2}, U_{1}\right)}{Q\left(U_{1}, U_{2}\right)}$

olup buna $\Pi$ nin kesit eğriliği denir ve $K(\Pi)$ ile gösterilir.

$\forall p \in M$ ve $U_{1_{p}}, U_{2_{p}} \in T_{M}(p)$ için, $K\left(U_{1_{p}}, U_{2_{p}}\right)$ sabit ise $M$ ye sabit kesit eğrilikli uzay form veya reel uzay form denir.

Tanım 2.6. $M$ Riemann manifoldu reel bir uzay form ve $c$-sabit kesit eğrilikli ise $M$ nin Riemann eğrilik tensörü

$$
R\left(U_{1}, U_{2}\right) U_{3}=c\left\{g\left(U_{2}, U_{3}\right) U_{1}-g\left(U_{1}, U_{3}\right) U_{2}\right\}
$$

şeklindedir (Yano ve Kon, 1984). 
$M, n$-boyutlu diferansiyellenebilir bir manifold olmak üzere $M$ üzerinde her bir $U_{1}, U_{2}$ vektör alanı için, $\xi$, bir kontravaryant vektör alanı, $\eta$ bir 1-form ve $(1,1)$-tipinden bir tensör alanı $\phi$ olmak üzere

$\phi^{2} U_{1}=U_{1}-\eta\left(U_{1}\right) \xi, \phi \xi=0, \eta\left(\phi U_{1}\right)=0, \eta(\xi)=1$

ve

$g\left(\phi U_{1}, \phi U_{2}\right)=g\left(U_{1}, U_{2}\right)-\eta\left(U_{1}\right) \eta\left(U_{2}\right), \quad \eta\left(U_{1}\right)=g\left(U_{1}, \xi\right)$,

eşitliklerini sağlayan $(M, \phi, \xi, \eta, g)$ beşlisine bir hemen hemen parakontakt metrik manifold denir (Sato, 1976). Bir hemen hemen parakontakt metrik manifoldu

$\left(\nabla_{U_{1}} \phi\right) U_{2}=-g\left(U_{1}, U_{2}\right) \xi-\eta\left(U_{2}\right) U_{1}+2 \eta\left(U_{1}\right) \eta\left(U_{2}\right) \xi$

ve

$\phi U_{1}=\nabla_{U_{1}} \xi$

eşitliklerini de sağlıyorsa bu durumda manifolda normal hemen hemen parakontakt metrik manifold denir.

Ayrıca $c$-sabit kesit eğriliğine sahip bir normal hemen hemen parakontakt metrik manifoldun Riemann eğrilik tensörü $R, \forall U_{1}, U_{2}, U_{3} \in \chi(M)$ olmak üzere

$$
\begin{aligned}
R\left(U_{1}, U_{2}\right) U_{3}= & \frac{c+3}{4}\left\{g\left(U_{2}, U_{3}\right) U_{1}-g\left(U_{1}, U_{3}\right) U_{2}\right\} \\
+ & \frac{c-1}{4}\left\{\eta\left(U_{1}\right) \eta\left(U_{3}\right) U_{2}-\eta\left(U_{2}\right) \eta\left(U_{3}\right) U_{1}+g\left(U_{1}, U_{3}\right) \eta\left(U_{2}\right) \xi-g\left(U_{2}, U_{3}\right) \eta\left(U_{1}\right) \xi\right. \\
& \left.+g\left(\phi U_{2}, U_{3}\right) \phi U_{1}-g\left(\phi U_{1}, U_{3}\right) \phi U_{2}-2 g\left(\phi U_{1}, U_{2}\right) \phi U_{3}\right\}
\end{aligned}
$$

ile verilir (Sato, 1976).

$n$-boyutlu bir Riemann manifoldunun projektif eğrilik tensörü, concircular eğrilik tensörüne quasi konformal eğrilik tensörü sirasıyla

$$
\begin{aligned}
P\left(U_{1}, U_{2}\right) U_{3}= & R\left(U_{1}, U_{2}\right) U_{3}-\frac{1}{n-1}\left[S\left(U_{2}, U_{3}\right) U_{1}-S\left(U_{1}, U_{3}\right) U_{2}\right] \\
\tilde{Z}\left(U_{1}, U_{2}\right) U_{3}= & R\left(U_{1}, U_{2}\right) U_{3}-\frac{r}{n(n-1)}\left[g\left(U_{2}, U_{3}\right) U_{1}-g\left(U_{1}, U_{3}\right) U_{2}\right] \\
\tilde{C}\left(U_{1}, U_{2}\right) U_{3}= & a R\left(U_{1}, U_{2}\right) U_{3}+b\left[S\left(U_{2}, U_{3}\right) U_{1}-S\left(U_{1}, U_{3}\right) U_{2}+g\left(U_{2}, U_{3}\right) Q U_{1}-g\left(U_{1}, U_{3}\right) Q U_{2}\right] \\
& -\frac{r}{n}\left[\frac{a}{n-1}+2 b\right]\left[g\left(U_{2}, U_{3}\right) U_{1}-g\left(U_{1}, U_{3}\right) U_{2}\right]
\end{aligned}
$$

şeklinde tanımlıdır. Burada $a$ ve $b$ birer sabit, $Q$, Ricci operatörü, $S$, Ricci tensörü ve $r$ manifoldun skaler eğriliğidir.

$M, n$-boyutlu bir normal hemen hemen parakontakt metrik manifoldu ve $M$ nin Riemann eğrilik tensörü $R$ olmak üzere, (5) denkleminde $U_{1}=\xi$ seçtiğimizde

$R\left(\xi, U_{2}\right) U_{3}=g\left(U_{2}, U_{3}\right) \xi-\eta\left(U_{3}\right) U_{2}$

benzer şekilde (5) de $U_{3}=\xi$ seçtiğimizde

$R\left(U_{1}, U_{2}\right) \xi=\eta\left(U_{2}\right) U_{1}-\eta\left(U_{1}\right) U_{2}$,

$R\left(U_{1}, \xi\right) \xi=U_{1}-\eta\left(U_{1}\right) \xi$ 
elde edilir. Ayrıca (5) denkleminde eşitliğin her iki tarafına $\xi \in \chi(M)$ uyguladığımızda ise

$\eta\left(R\left(U_{1}, U_{2}\right) U_{3}\right)=g\left(U_{2}, U_{3}\right) \eta\left(U_{1}\right)-g\left(U_{1}, U_{3}\right) \eta\left(U_{2}\right)$

eşitliği hesaplanır. Benzer şekilde (6), (7) ve (8) denklemlerinden,

$P\left(\xi, U_{2}\right) U_{3}=g\left(U_{2}, U_{3}\right) \xi-\frac{1}{n-1} S\left(U_{2}, U_{3}\right) \xi$,

$P\left(\xi, U_{2}\right) \xi=0$,

$\tilde{Z}\left(\xi, U_{2}\right) U_{3}=\left[1-\frac{r}{n(n-1)}\right]\left[g\left(U_{2}, U_{3}\right) \xi-\eta\left(U_{3}\right) U_{2}\right]$,

$\tilde{Z}\left(\xi, U_{2}\right) \xi=\left[1-\frac{r}{n(n-1)}\right]\left[\eta\left(U_{2}\right) \xi-U_{2}\right]$,

$\tilde{C}\left(\xi, U_{2}\right) U_{3}=\left[\frac{4 a+b(n-5)(1-c)}{4}-\frac{r}{n}\left[\frac{a}{n-1}+2 b\right]\right]\left[g\left(U_{2}, U_{3}\right) \xi-\eta\left(U_{3}\right) U_{2}\right]$,

$\tilde{C}\left(\xi, U_{2}\right) \xi=\left[\frac{4 a+b(n-5)(1-c)}{4}-\frac{r}{n}\left[\frac{a}{n-1}+2 b\right]\right]\left[\eta\left(U_{2}\right) \xi-U_{2}\right]$

eşitlikleri elde edilir.

Ayrıca burada $M \operatorname{nin}\left\{e_{1}, e_{2}, \ldots, e_{n-1}, \xi\right\}$ bazı için

$S\left(U_{1}, U_{2}\right)=\sum_{\mathrm{i}=1}^{\mathrm{n}} g\left(R\left(U_{1}, e_{i}\right) e_{i}, U_{2}\right)+g\left(R\left(U_{1}, \xi\right) \xi, U_{2}\right)$

olduğundan ve eşitlik (5) den

$$
\begin{aligned}
R\left(U_{1}, e_{i}\right) e_{i} & =\frac{c+1}{4}\left\{g\left(e_{i}, e_{i}\right) U_{1}-g\left(U_{1}, e_{i}\right) e_{i}\right\} \\
& +\frac{c-1}{4}\left\{-g\left(e_{i}, e_{i}\right) \eta\left(U_{1}\right) \xi+g\left(\phi\left(e_{i}\right), e_{i}\right) \phi\left(U_{1}\right)-3 g\left(\phi\left(U_{1}\right), e_{i}\right) \phi e_{i}\right\}
\end{aligned}
$$

olduğundan

$S\left(U_{1}, U_{2}\right)=\left[\frac{c(n-5)+3 n+1}{4}\right] g\left(U_{1}, U_{2}\right)+\left[\frac{(c-1)(5-n)}{4}\right] \eta\left(U_{1}\right) \eta\left(U_{2}\right)$

ve

$Q U_{1}=\left[\frac{c(n-5)+3 n+1}{4}\right] U_{1}+\left[\frac{(c-1)(5-n)}{4}\right] \eta\left(U_{1}\right) \xi$

elde edilir.

Sonuç 2.1. Her sabit kesit eğrilikli normal hemen hemen parakontakt metrik manifold $\eta$-Einstein manifolddur.

Ayrıca (19) denkleminde $U_{2}=\xi$ seçtiğimizde

$S\left(U_{1}, \xi\right)=(n-1) \eta\left(U_{1}\right)$,

benzer şekilde

$Q \xi=(n-1) \xi$

olarak hesaplanır. Diğer taraftan $M$ nin skaler eğriliği 
$r=\frac{(n-1)}{4}[c(n-5)+3 n+5]$

dir.

\section{Bir Normal Hemen Hemen Parakontakt Metrik Manifoldun Quasi-Konformal Ĕgrilik Tensörü}

Bu bölümde sabit kesit eğrilikli bir normal hemen hemen parakontakt metrik manifoldun quasi-konformal eğrilik tensörünün diğer eğrilik tensörleri üzerindeki etkiler incelenmiştir.

Teorem 3.1. $n$-boyutlu bir $M(c)$-sabit kesit eğrilikli normal hemen hemen parakontakt metrik manifoldunda $\tilde{C}\left(\xi, U_{2}\right) R=0$ olması için gerek ve yeter şart ya manifold $M(1)$ şeklinde bir reel uzay formdur yada manifoldun skaler eğriliği

$r=\frac{n(n-1)[4 a+b(n-5)(1-c)]}{4 a+8 b(n-1)}$

dir.

Ispat: Kabul edelim ki $\tilde{C}\left(\xi, U_{2}\right) R=0$ şartı sağlansın. $\mathrm{O}$ zaman $\forall U_{1}, U_{2}, U_{3}, U_{4}, U_{5} \in \chi(M)$ olmak

$$
\begin{aligned}
\left(\tilde{C}\left(U_{1}, U_{2}\right) R\right)\left(U_{3}, U_{4}, U_{5}\right)= & \tilde{C}\left(U_{1}, U_{2}\right) R\left(U_{3}, U_{4}\right) U_{5}-R\left(\tilde{C}\left(U_{1}, U_{2}\right) U_{3}, U_{4}\right) U_{5} \\
& -R\left(U_{3}, \tilde{C}\left(U_{1}, U_{2}\right) U_{4}\right) U_{5}-R\left(U_{3}, U_{4}\right) \tilde{C}\left(U_{1}, U_{2}\right) U_{5}
\end{aligned}
$$

olduğunu biliyoruz. (24) denkleminde $U_{1}=\xi$ seçtiğimizde

$$
\begin{aligned}
\left(\tilde{C}\left(\xi, U_{2}\right) R\right)\left(U_{3}, U_{4}, U_{5}\right) & =\tilde{C}\left(\xi, U_{2}\right) R\left(U_{3}, U_{4}\right) U_{5}-R\left(\tilde{C}\left(\xi, U_{2}\right) U_{3}, U_{4}\right) U_{5} \\
& -R\left(U_{3}, \tilde{C}\left(\xi, U_{2}\right) U_{4}\right) U_{5}-R\left(U_{3}, U_{4}\right) \tilde{C}\left(\xi, U_{2}\right) U_{5} \\
& =0
\end{aligned}
$$

halini olur. Bu denklemde (17) kullanıldığında

$$
\begin{aligned}
{\left[\frac{4 a+b(n-5)(1-c)}{4}-\frac{r}{n}\left[\frac{a}{n-1}+2 b\right]\right] } & {\left[g\left(U_{2}, R\left(U_{3}, U_{4}\right) U_{5}\right) \xi-\eta\left(R\left(U_{3}, U_{4}\right) U_{5}\right) U_{2}\right.} \\
& -g\left(U_{2}, U_{3}\right) R\left(\xi, U_{4}\right) U_{5}+\eta\left(U_{3}\right) R\left(U_{2}, U_{4}\right) U_{5} \\
& -g\left(U_{2}, U_{4}\right) R\left(U_{3}, \xi\right) U_{5}+\eta\left(U_{4}\right) R\left(U_{3}, U_{2}\right) U_{5} \\
& \left.-g\left(U_{2}, U_{5}\right) R\left(U_{3}, U_{4}\right) \xi+\eta\left(U_{5}\right) R\left(U_{3}, U_{4}\right) U_{2}\right] \\
& =0
\end{aligned}
$$

bulunur. (26) da (9) ve (10) eşitlikleri kullanıldığında

$\left[\frac{4 a+b(n-5)(1-c)}{4}-\frac{r}{n}\left[\frac{a}{n-1}+2 b\right]\right]\left[R\left(U_{2}, U_{4}\right) U_{5}+g\left(U_{2}, U_{5}\right) U_{4}-g\left(U_{4}, U_{5}\right) U_{2}\right]=0$

elde edilir. Buradan ya

$R\left(U_{2}, U_{4}\right) U_{5}=g\left(U_{4}, U_{5}\right) U_{2}-g\left(U_{2}, U_{5}\right) U_{4}$

için $M, c=1$ sabit kesit eğrilikli bir reel uzay formdur ya da $M$ nin skaler eğriliği

$r=\frac{n(n-1)[4 a+b(n-5)(1-c)]}{4 a+8 b(n-1)}$

dir. İspatın tersi açıtır. 
Teorem 3.2. $n$-boyutlu bir $M(c)$-sabit kesit eğrilikli normal hemen hemen parakontakt metrik manifoldunda $\tilde{C}\left(\xi, U_{2}\right) P=0$ olması için gerek ve yeter şart ya $M$ bir Einstein manifoldudur ya da $M$ nin skaler eğriliği

$r=\frac{n(n-1)[4 a+b(n-5)(1-c)]}{4 a+8 b(n-1)}$

dir.

Ispat. Kabul edelim ki $\tilde{C}\left(\xi, U_{2}\right) P=0$ şartı sağlansın. $\mathrm{O}$ zaman $\forall U_{2}, U_{3}, U_{4}, U_{5} \in \chi(M)$ olmak üzere

$$
\begin{aligned}
\left(\tilde{C}\left(\xi, U_{2}\right) P\right)\left(U_{3}, U_{4}, U_{5}\right) & =\tilde{C}\left(\xi, U_{2}\right) P\left(U_{3}, U_{4}\right) U_{5}-P\left(\tilde{C}\left(\xi, U_{2}\right) U_{3}, U_{4}\right) U_{5} \\
& -P\left(U_{3}, \tilde{C}\left(\xi, U_{2}\right) U_{4}\right) U_{5}-P\left(U_{3}, U_{4}\right) \tilde{C}\left(\xi, U_{2}\right) U_{5} \\
& =0
\end{aligned}
$$

dir. (28) denkleminde (17) kullanılırsa

$$
\begin{aligned}
{\left[\frac{4 a+b(n-5)(1-c)}{4}-\frac{r}{n}\left[\frac{a}{n-1}+2 b\right]\right] } & {\left[g\left(U_{2}, P\left(U_{3}, U_{4}\right) U_{5}\right) \xi-\eta\left(P\left(U_{3}, U_{4}\right) U_{5}\right) U_{2}\right.} \\
& -g\left(U_{2}, U_{3}\right) P\left(\xi, U_{4}\right) U_{5}+\eta\left(U_{3}\right) P\left(U_{2}, U_{4}\right) U_{5} \\
& -g\left(U_{2}, U_{4}\right) P\left(U_{3}, \xi\right) U_{5}+\eta\left(U_{4}\right) P\left(U_{3}, U_{2}\right) U_{5} \\
& \left.-g\left(U_{2}, U_{5}\right) P\left(U_{3}, U_{4}\right) \xi+\eta\left(U_{5}\right) P\left(U_{3}, U_{4}\right) U_{2}\right]=0
\end{aligned}
$$

bulunur. (29) da (13) ve (14) eşitlikleri kullanıldığında ve sonrasında $U_{3}=\xi$ seçtiğimizde

$$
\begin{aligned}
& {\left[\frac{4 a+b(n-5)(1-c)}{4}-\frac{r}{n}\left[\frac{a}{n-1}+2 b\right]\right]\left[P\left(U_{2}, U_{4}\right) U_{5}+g\left(U_{4}, U_{2}\right) \eta\left(U_{5}\right) \xi\right.} \\
&-\frac{1}{n-1} g\left(U_{4}, U_{2}\right) \eta\left(U_{5}\right) \xi+g\left(U_{2}, U_{5}\right) \eta\left(U_{4}\right) \xi-\frac{1}{n-1} g\left(U_{2}, U_{5}\right) \eta\left(U_{4}\right) \xi \\
&+\frac{1}{n-1} S\left(U_{4}, U_{5}\right) U_{2}-g\left(U_{4}, U_{5}\right) U_{2}-\frac{1}{n-1} S\left(U_{2}, U_{5}\right) \eta\left(U_{4}\right) \xi \\
&\left.+\frac{1}{n-1} g\left(U_{2}, U_{5}\right) U_{4}-\frac{1}{n-1} S\left(U_{2}, U_{4}\right) \eta\left(U_{5}\right) \xi\right] \\
&=0
\end{aligned}
$$

elde edilir. (30) denkleminde $U_{5}=\xi$ seçtiğimizde ve gerekli kısaltmaları yaptığımızda

$$
\left[\frac{4 a+b(n-5)(1-c)}{4}-\frac{r}{n}\left[\frac{a}{n-1}+2 b\right]\right]\left[\frac{1}{n-1} S\left(U_{4}, U_{2}\right)-g\left(U_{4}, U_{2}\right)\right]=0
$$

bulunur. Buradan ya $M$ nin skaler eğriliği

$\mathrm{r}=\frac{n(n-1)[4 a+b(n-5)(1-c)]}{4 a+8 b(n-1)}$

olarak hesaplanır. Diğer taraftan

$S\left(U_{4}, U_{2}\right)=(n-1) g\left(U_{4}, U_{2}\right)$

olup buradan $M$ bir Einstein manifoldudur. İspatın tersi açıktır. 
Teorem 3.3. $n$-boyutlu bir $M(c)$-sabit kesit eğrilikli normal hemen hemen parakontakt metrik manifoldunda $\tilde{C}\left(\xi, U_{2}\right) \tilde{Z}=0$ olması için gerek ve yeter şart ya $M, c=1$ sabit kesit eğrilikli bir reel uzay formdur ya da $M$ nin skaler eğriliği

$r=\frac{n(n-1)[4 a+b(n-5)(1-c)]}{4 a+8 b(n-1)}$

dir.

Ispat: Kabul edelim ki $\tilde{C}\left(\xi, U_{2}\right) \tilde{Z}=0$ olsun. $\mathrm{O}$ zaman $\forall U_{2}, U_{3}, U_{4}, U_{5} \in \chi(M)$ olmak üzere

$$
\begin{aligned}
&\left(\tilde{C}\left(\xi, U_{2}\right) \tilde{Z}\right)\left(U_{3}, U_{4}, U_{5}\right)=\tilde{C}\left(\xi, U_{2}\right) \tilde{Z}\left(U_{3}, U_{4}\right) U_{5}-\tilde{Z}\left(\tilde{C}\left(\xi, U_{2}\right) U_{3}, U_{4}\right) U_{5} \\
&-\tilde{Z}\left(U_{3}, \tilde{C}\left(\xi, U_{2}\right) U_{4}\right) U_{5}-\tilde{Z}\left(U_{3}, U_{4}\right) \tilde{C}\left(\xi, U_{2}\right) U_{5} \\
&=0
\end{aligned}
$$

dir. (31) denkleminde (17) kullanılırsa

$$
\begin{gathered}
{\left[\frac{4 a+b(n-5)(1-c)}{4}-\frac{r}{n}\left[\frac{a}{n-1}+2 b\right]\right]\left[g\left(U_{2}, \tilde{Z}\left(U_{3}, U_{4}\right) U_{5}\right) \xi-\eta\left(\tilde{Z}\left(U_{3}, U_{4}\right) U_{5}\right) U_{2}\right.} \\
-g\left(U_{2}, U_{3}\right) \tilde{Z}\left(\xi, U_{4}\right) U_{5}+\eta\left(U_{3}\right) \tilde{Z}\left(U_{2}, U_{4}\right) U_{5} \\
-g\left(U_{2}, U_{4}\right) \tilde{Z}\left(U_{3}, \xi\right) U_{5}+\eta\left(U_{4}\right) \tilde{Z}\left(U_{3}, U_{2}\right) U_{5} \\
\left.-g\left(U_{2}, U_{5}\right) \tilde{Z}\left(U_{3}, U_{4}\right) \xi+\eta\left(U_{5}\right) \tilde{Z}\left(U_{3}, U_{4}\right) U_{2}\right] \\
=0
\end{gathered}
$$

elde edilir. (32) denkleminde (15) ve (16) eşitlikleri kullanıldığında ve sonrasında $U_{3}=\xi$ seçtiğimizde

$$
\begin{gathered}
{\left[\frac{4 a+b(n-5)(1-c)}{4}-\frac{r}{n}\left[\frac{a}{n-1}+2 b\right]\right]\left[\tilde{Z}\left(U_{2}, U_{4}\right) U_{5}\right.} \\
\left.-\left[1-\frac{r}{n(n-1)}\right]\left[g\left(U_{4}, U_{5}\right) U_{2}-g\left(U_{2}, U_{5}\right) U_{4}\right]\right] \\
=0
\end{gathered}
$$

elde edilir. (33) denkleminde (7) kullanılırsa ya $M, c=1$ sabit kesit eğrilikli bir reel uzay formdur ya da $M$ nin skaler eğriliği

$\mathrm{r}=\frac{n(n-1)[4 a+b(n-5)(1-c)]}{4 a+8 b(n-1)}$

dir. Böylece ispat tamamlanır, tersi ise açıktır.

Teorem 3.4. $\mathrm{n}$-boyutlu bir $M(c)$-sabit kesit eğrilikli normal hemen hemen parakontakt metrik manifoldunda $\tilde{\mathrm{C}}\left(\xi, U_{2}\right) \mathrm{S}=0$ olması için gerek ve yeter şart ya $\mathrm{M}$ bir Einstein manifoldudur ya da manifoldun skaler eğriliği

$r=\frac{n(n-1)[4 a+b(n-5)(1-c)]}{4 a+8 b(n-1)}$

dir.

Ispat: Kabul edelim ki $\tilde{C}\left(\xi, U_{2}\right) S=0$ olsun. O zaman $\forall U_{1}, U_{2}, U_{3}, U_{4} \in \chi(M)$ için

$S\left(\tilde{C}\left(U_{1}, U_{2}\right) U_{3}, U_{4}\right)+S\left(U_{3}, \tilde{C}\left(U_{1}, U_{2}\right) U_{4}\right)=0$

dir. (34) denkleminde $U_{1}=\xi$ seçtiğimizde 


$$
\begin{gathered}
{\left[\frac{4 a+b(n-5)(1-c)}{4}-\frac{r}{n}\left[\frac{a}{n-1}+2 b\right]\right]\left[(n-2) g\left(U_{2}, U_{3}\right) \eta\left(U_{4}\right)+(n-2) g\left(U_{2}, U_{4}\right) \eta\left(U_{3}\right)\right.} \\
\left.-\eta\left(U_{3}\right) S\left(U_{2}, U_{4}\right)-\eta\left(U_{4}\right) S\left(U_{3}, U_{2}\right)\right] \\
=0
\end{gathered}
$$

bulunur. Buradan ya

$S\left(U_{2}, U_{4}\right)=(n-1) g\left(U_{2}, U_{4}\right)$

olup buradan $M$ bir Einstein manifoldudur. Ya da

$r=\frac{n(n-1)[4 a+b(n-5)(1-c)]}{4 a+8 b(n-1)}$

dir. İspat tamamlanır, tersi açıcça görülür.

Teorem 3.5. $n$-boyutlu bir $M(c)$ sabit kesit eğrilikli normal hemen hemen parakontakt metrik manifoldunda $\tilde{\mathrm{C}}\left(\xi, U_{2}\right) \tilde{\mathrm{C}}=0$ olması için gerek ve yeter şart ya $M\left(\frac{4 a-b[5 n-7-c(3 n+5)]}{4 a}\right)$ sabit kesit eğrilikli bir reel uzay form ya da $M$ nin skaler eğriliği

$r=\frac{n(n-1)[4 a+b(n-5)(1-c)]}{4 a+8 b(n-1)}$

dir.

Ispat: Kabul edelim ki $\tilde{C}\left(\xi, U_{2}\right) \tilde{C}=0$ olsun. $\mathrm{O}$ zaman $\forall U_{2}, U_{3}, U_{4}, U_{5} \in \chi(M)$ olmak üzere

$$
\begin{gathered}
\left(\tilde{C}\left(\xi, U_{2}\right) \tilde{C}\right)\left(U_{4}, U_{5}, U_{3}\right)=\tilde{C}\left(\xi, U_{2}\right) \tilde{C}\left(U_{4}, U_{5}\right) U_{3}-\tilde{C}\left(\tilde{C}\left(\xi, U_{2}\right) U_{4}, U_{5}\right) U_{3} \\
-\tilde{C}\left(U_{4}, \tilde{C}\left(\xi, U_{2}\right) U_{5}\right) U_{3}-\tilde{C}\left(U_{4}, U_{5}\right) \tilde{C}\left(\xi, U_{2}\right) U_{3} \\
=0
\end{gathered}
$$

dir. (36) eşitliğinde (17) denklemi kullanıldığında

$$
\begin{array}{r}
{\left[\frac{4 a+b(n-5)(1-c)}{4}-\frac{r}{n}\left[\frac{a}{n-1}+2 b\right]\right][} \\
{\left[g\left(U_{2}, \tilde{C}\left(U_{4}, U_{5}\right) U_{3}\right) \xi-\eta\left(\tilde{C}\left(U_{4}, U_{5}\right) U_{3}\right) U_{2}\right.} \\
-g\left(U_{2}, U_{4}\right) \tilde{C}\left(\xi, U_{5}\right) U_{3}+\eta\left(U_{4}\right) \tilde{C}\left(U_{2}, U_{5}\right) U_{3} \\
-g\left(U_{2}, U_{5}\right) \tilde{C}\left(U_{4}, \xi\right) U_{3}+\eta\left(U_{5}\right) \tilde{C}\left(U_{4}, U_{2}\right) U_{3} \\
\left.-g\left(U_{2}, U_{3}\right) \tilde{C}\left(U_{4}, U_{5}\right) \xi+\eta\left(U_{3}\right) \tilde{C}\left(U_{4}, U_{5}\right) U_{2}\right] \\
=0
\end{array}
$$

elde edilir. (37) denkleminde tekrar (17) kullanılırsa ve $U_{4}=\xi$ seçildiğinde,

$$
\begin{aligned}
& {\left[\frac{4 a+b(n-5)(1-c)}{4}-\frac{r}{n}\left[\frac{a}{n-1}+2 b\right]\right]\left[\tilde{C}\left(U_{2}, U_{5}\right) U_{3}\right.} \\
& \left.\left.-\left[\frac{4 a+b(n-5)(1-c)}{4}-\frac{r}{n}\left[\frac{a}{n-1}+2 b\right]\right]\left[g\left(U_{5}, U_{3}\right) U_{2}-g\left(U_{2}, U_{3}\right) U_{5}\right)\right]\right] \\
& =0
\end{aligned}
$$

eşitliği bulunur. Son eşitlikte $U_{2} \rightarrow \phi U_{2}$ ve $U_{5} \rightarrow \phi U_{5}$ seçilip, sonrasında (8) kullanıldığında 
$R\left(\phi U_{2}, \phi U_{5}\right) U_{3}=\left[\frac{4 a-b[5 n-7-c(3 n+5)]}{4 a}\right]\left[g\left(\phi U_{5}, U_{3}\right) \phi U_{2}-g\left(\phi U_{2}, U_{3}\right) \phi U_{5}\right]$

olarak hesaplanır. (38) eşitliğinden ise $M\left(\frac{4 a-b[5 n-7-c(3 n+5)]}{4 a}\right)$ sabit kesit eğrilikli bir reel uzay formdur.

Diğer taraftan $M$ nin skaler eğriliğinin de

$r=\frac{n(n-1)[4 a+b(n-5)(1-c)]}{4 a+8 b(n-1)}$

olduğu görülür. İspatın tersi ise açıktır.

Örnek 3.1. $\mathbb{R}^{7}$ de $\left(x_{1}, x_{2}, x_{3}, y_{1}, y_{2}, y_{3}, z\right)$ standart koordinatlarına göre $M^{7}=\left\{\left(x_{1}, x_{2}, x_{3}, y_{1}, y_{2}, y_{3}, z\right) \in\right.$ $\left.\mathbb{R}^{7}\right\}$ manifoldunu alalım. $M$ nin her bir noktasında lineer bağımsız vektör alanları

$e_{i}=e^{z} \frac{\partial}{\partial x_{i}}, \quad e_{j}=e^{z} \frac{\partial}{\partial y_{j}}, \quad 1 \leq i, j \leq 3$ ve $e_{7}=\frac{\partial}{\partial z}$

olsun. Burada $M$ üzerinde Riemann metriği

$g=e^{-2 z} \sum_{i=1}^{3}\left\{d x_{i} \otimes d y_{i}+d y_{i} \otimes d y_{i}\right\}+d z \otimes d z$

olsun. Burada $g\left(e_{i}, e_{j}\right)=\delta_{i j}$ olup, $1 \leq i, j \leq 7$ olmak üzere $e_{i}, M$ nin bir ortonormal bazıdır. $M$ üzerinde bir vektör alanı

$X=\sum_{i=1}^{3}\left(X_{i} \frac{\partial}{\partial x_{i}}+Y_{i} \frac{\partial}{\partial y_{i}}\right)+z \frac{\partial}{\partial z}$

dir. Burada $\phi$ parakontakt yapısını ve $\eta 1$-formunu ise

$\phi X=\sum_{i=1}^{3}\left(-X_{i} \frac{\partial}{\partial x_{i}}-Y_{i} \frac{\partial}{\partial y_{i}}\right)$

ve

$\eta(X)=g\left(X, e_{7}\right)$ olarak tanımlayalım. Bundan kolayca görülebilir ki her $X, Y \in \Gamma(T M)$ için

$\phi^{2} X=X-\eta(X) e_{7}$,

$g(\phi X, \phi Y)=g(X, Y)-\eta(X) \eta(Y)$

ve

$\eta\left(e_{7}\right)=1$

dir. Böylece $\left(\phi, \xi=e_{7}, \eta, g\right) M$ üzerinde bir hemen hemen parakontakt metrik yapıdır. Burada Lie parantez operatörleri de

$\left[e_{i}, e_{7}\right]=-e_{i}, \quad 1 \leq i \leq 6$ ve $\left[e_{i}, e_{j}\right]=0,1 \leq j \leq 6$

olarak hesaplanır.

Yine Kozsul formülü yardımıyla kovaryant türevler ise

$\nabla_{e_{i}} e_{i}=e_{7}, \quad \nabla_{e_{i}} e_{j}=0, \quad i \neq j, \quad 1 \leq i, j \leq 6$,

$\nabla_{e_{i}} e_{7}=\phi e_{i}=-e_{i}, \quad \nabla_{e_{7}} e_{7}=0, \quad, \nabla_{e_{7}} e_{i}=0 \quad 1 \leq i \leq 6$ 
olarak bulunur. Yine burada $X, Y \in \Gamma(T M)$ için

$\left(\nabla_{X} \phi\right) Y=-g(X, Y) \xi-\eta(Y) X+2 \eta(X) \eta(Y) \xi$

olduğu görülür. Böylece $M^{7}(\phi, \xi, \eta, g)$ yapısı bir hemen hemen normal parakontakt metrik manifolddur. $M$ nin Riemann eğrilik tensörü yardımıyla,

$R\left(e_{i}, e_{j}\right) e_{j}=-e_{i}, \quad 1 \leq i \neq j \leq 7$,

$R\left(e_{i}, e_{j}\right) e_{k}=0, \quad 1 \leq i, j, k \leq 6, \quad i \neq j \neq k$

değerleri bulunur. Şimdi $M$ üzerindeki $X, Y, Z$ vektör alanları

$X=X_{i} e_{i}, \quad Y=Y_{j} e_{j} \quad$ ve $Z=Z_{k} e_{k}, \quad 1 \leq i, j, k \leq 7$ olarak alırsak,

$$
\begin{aligned}
R(X, Y) Z & =X_{i} Y_{j} Z_{k} R\left(e_{i}, e_{j}\right) e_{k}=Y_{j} Z_{j} X_{i} R\left(e_{i}, e_{j}\right) e_{j}+X_{i} Y_{j} Z_{i} R\left(e_{i}, e_{j}\right) e_{i} \\
& =Y_{j} Z_{j} X_{i} e_{i}+X_{i} Z_{i} Y_{j} e_{j} \\
& =-\{g(Y, Z) X-g(X, Z) Y\}
\end{aligned}
$$

dir. Yani $M \quad c=-1$ sabit kesit eğriliklidir. Buradan da $M$ nin Ricci tensörü

$S(X, Y)=-(n-1) g(X, Y)$

olduğundan

$S(X, Y)=-6 g(X, Y)$ dir. Buradan da $M$ nin skaler eğriliği $r=-42$ olarak hesaplanır.

Sonuç olarak verdiğimiz bütün teoremleri sağlayan örneğimiz için $c=-1, r=-42$ olup ayrıca $a=-\frac{13}{2} b$ bağıntısı vardır.

\section{Kaynaklar}

Acet, B.E. ve Perktaş, S.Y., 2016. On para-Sasakian manifolds with a canonical paracontact connection, New Trends in Math. Sci. 4, No. 3, 162-173, 2016.

Acet, B.E., Kılıç, E. ve Perktaş, S.Y., 2012, Some Curvature Conditions on a Para-Sasakian Manifold with Canonical Paracontact Connection, International Journal of Mathematics and Mathematical Sciences, Volume 2012, Article ID 395462, 24 pages.

Atçeken, M. ve Yıldırım, Ü., 2016. On an almost $C(\alpha)$-manifolds satisfying certain conditions on quasi-conformal curvature tensor. Proceedings of the Jangjeon Mathematical Society, 19 (1), 115-124.

Atçeken, M., 2013. Some curvature properties of $(L C S)_{n}$-manifolds. Abstract and Applied Analysis, Doi: 10.1055/2013/380657.
Atçeken, M., Dirik, S. ve Yıldırım, Ü., 2017. An Inequality for Warped Product Semi-Invariant Submanifolds of a Normal Paracontact Metric Manifold. Filomat 31:19, 6233-6240.

Atçeken, M., Yıldırım, Ü. ve Dirik, S., 2017. SubManifolds of a Riemannian Manifold. Manifolds: Current Research Areas, Prof. Paul Bracken, InTech, DOI: 10.5772/65948.

Boothby, W.M., 1986. An Introduction to Differentiable Manifolds and Riemanniann Geometry. Academic Press, Inc. London.

De, U.C., Jun, J.B., ve Gazi, A.K., 2008. Sasakian manifolds with quasi-conformal curvature tensor. Bulletin of the Korean Mathematical Society, 45 (2) 313-319.

De, U.C., ve Mondal, A.K., 2009. On 3-dimensional normal almost contact metric manifolds satisfying certain curvature conditions. Commun. Korean. Math. Soc., 24, 265-275. 
De, U.C., ve Sarkar, A., 2012. On the quasi-conformal curvature tensor of $(\kappa, \mu)$-contact metric manifold. Mathematical Reports, 14 (2) 115129.

De, U.C., Yıldız, A., ve Yalınız, A.F., 2009. Locally $\phi$-symmetric normal almost contact metric manifolds of dimension 3. Appl. Math. Lett., 22, 723-727.

Erdem, S., 2002. On almost (para)contact (hyperbolic) metric manifolds and harmonicity of $\left(\phi, \phi^{\prime}\right)$-holomorphic maps between them. Houston J. Math., 28, 21-45.

Erken, İ.K., 2015. On normal almost paracontact metric manifolds of dimension 3. Facta Universitatis (NIS), Ser. Math. Inform., 30 (5) 777-788

Hosseinzadeh, A., ve Taleshian, A., 2012. On conformal and quasi conformal curvature tensors of an $N(k)$-quasi Einstein manifold. Communucations of the KMS, 27 (2), 317-326.

Kaneyuki, S., ve Williams, F.L., 1985. Almost paraccontact and parahodge structures on manifolds. Nagoya Math. J., 99, 173-187.

Martin, M.V., 2015. On a remarkable class of paracontact metric manifolds. International Journal of Geometric Methods in Modern Physics, 12 (8).
O’Neill, B., 1983. Semi-Riemann Geometry with Applications to Relativity. Pure and Applied Mathematics, 103, Academ,c Press, Inc. New York.

Olszak, Z., 1986. Normal almost contact metric manifolds of dimension three. Ann. Polon. Math., XLVII, 41-50.

Pandey, H.B. ve Kumar, A., 1985. Anti invariant submanifolds of almost paracontact manifolds. Indian J. Püre appl. Math., 16(6), 586-590.

Sato, I., 1976. On a structure similar to the almost contact structure, Tensor (N.S.), 30, no. 3, 219 224.

Sato, I., 1977. On a structure similar to almost contact structures II, Tensor (N.S.), 31, no. 2, 199-205.

Szabo, Z.I., 1982. Structure theorems on Riemannian spaces satisfying $R(X, Y) R=0$ the local version. Diff. Geom., 17, 531-582.

Yano, K., ve Kon, M., 1984. Structres on Manifolds. Series in Pure Mathematics, 3, World Scientific Publishing Co., Singapore, 72.

Yıldırım, Ü., Atçeken, M. ve Dirik, S. 2018. B-Y. Chen Inequalities for Semi-Slant Submanifolds in Normal Paracontact Metric Manifolds. Palestine Journal of Mathematics, Vol. 7(1)(2018) , 281-288. 